\title{
Effect of Selenium-yeast on Cognitive Performance on Pregnant Dams Exposed to Noise Stress
}

\author{
Okwute Michael Ochayi ${ }^{a, b *}$ Bond Anyaehie ${ }^{b}$, Eghosa lyare ${ }^{b}$ \\ DanAzumi Umar Haruna ${ }^{c}$ and Andrew E. Ivang
}

${ }^{a}$ Department of Physiology, Faculty of Basic Medical Sciences, College of Medicine and Health Sciences, Baze University, Abuja, Nigeria.

${ }^{b}$ Department of Physiology, Faculty of Basic Medical Sciences, College of Medicine and Health Sciences, University of Nigeria, Nsukka (Enugu Campus), Enugu State, Nigeria. ${ }^{c}$ Department of Anatomy, Faculty of Basic Medical Sciences, College of Medicine and Health Sciences, Baze University, Abuja, Nigeria.

${ }^{d}$ Department of Clinical Biology-Huye-Campus, Clinical Anatomy Unit, College of Medicine and Health Sciences, University of Rwanda, Kigali, Rwanda.

Authors' contributions

This work was carried out in collaboration among all authors. Authors OMO, BA and EI designed the study, performed the statistical analysis, wrote the protocol, and wrote the first draft of the manuscript.

Authors DAUH and AEI managed the literature searches. All authors read and approved the final manuscript.

Article Information

DOI: 10.9734/ARRB/2022/v37i130479

Open Peer Review History:

This journal follows the Advanced Open Peer Review policy. Identity of the Reviewers, Editor(s) and additional Reviewers, peer review comments, different versions of the manuscript, comments of the editors, etc are available here: https://www.sdiarticle5.com/review-history/81810

Original Research Article

Received 12 November 2021

Accepted 15 January 2022

Published 17 January 2022

\section{ABSTRACT}

A total of twelve pregnant Wistar rats were used, and randomly divided into three groups $(n=4)$. Group A (negative control), not exposed to noise stress), group B $(1 \mathrm{~mL} / \mathrm{kg}$ of distilled water $+4 \mathrm{~h}$ $/ 15$ days noise stress), group $C(0.04 \mathrm{mg} / \mathrm{kg})$ of selenium-yeast $+4 \mathrm{~h} / 15$ days noise stress). On day 18 and 19 of gestation cognitive tests were conducted using Y-maze and Novel Object Recognition Test. A significant increase $(P<0.05)$ was observed in the percentage alternation and discrimination index in group A compared with group B. However, the percentage alternation and DI were significantly $(P<0.05)$ decreased in group $C$ compared with group $B$. In conclusion, noise stress induces cognitive deficit, this deficit can be mitigated with the administration of seleniumyeast. 
Keywords: Noise stress; pregnant dams; cognition; selenium-yeast.

\section{INTRODUCTION}

Noise can be defined as the concomitant of unavoidable and unpleasant sounds that emerges from the environment in today's world via household appliances, worship centers, night clubs and urban traffic [1]. When noise exceeds $90 \mathrm{~dB}$ it's said to be a stress; which can induce harmful effects on the cardiovascular and central nervous systems [2]. Some studies have shown the adverse effects of noise stress on the brain as it induces behavioral changes and declines cognitive performance; with decrease in incidental, intentional and recognition memory in children [3,4]. A study conducted among industrial workers and individuals residing around major transports routes linked the occurrence of depression and aggression to noise exposure [4].

Since noise stress overwhelm the physiological adaptive mechanism by inducing excess secretion of glucocorticoids hormone in females when compared with males [5], and this secretion level has been found to increase during pregnancy; this may lead to a condition known as prenatal stress [6]. Prenatal stress has adverse effects on the structural and biochemical domain of fetal brain; by inhibiting neuronal development and might have possibly been the contributing factor to cognitive impairment [7]. One of the hypotheses of the effect of prenatal stress on the nervous system is due to the cortisol hormone crossing the blood-brain barrier, thereby inducing oxidative stress [8]. Since oxidative stress is the imbalance in the oxidants and antioxidants of the body; hence antioxidant is vital in mitigating the adverse effects of stress. Antioxidants are substances that inhibit oxidation caused by free-radicals in target cells; example of such is selenium which is a component of selenoenzymes [9]. Selenoenzymes are the families of glutathione peroxidases (GPXs), thioredoxin reductases (TRs) and iodothyronine deiodinases (Ds), and can be enriched with yeast to enhance potency [10].

However, most studies conducted on the impact of noise stress focused on the pubs while neglecting the pregnant dams, and also no effective therapy has been established in mitigating the resultant cognitive impairments. This study investigate the effects of seleniumyeast on the cognitive performance of the pregnant dams exposed to noise stress using object recognition and Y-maze test for cognitive performance measure.

\section{MATERIALS AND METHODS}

Twelve healthy female Wistar rats weighing 150$170 \mathrm{~g}$ were kept in the Animal House of Department of Physiology, Baze University Abuja, FCT-Abuja, Nigeria under standard laboratory conditions. The animals were divided randomly into three groups and a male rat was introduced to each group for mating. Pregnancy was confirmed by the presence of vaginal plug which was tagged pregnancy day zero (PDO). The rats were grouped as follow: group $A-$ Negative Control (NC) (not exposed to noise +1 $\mathrm{mL} / \mathrm{kg}$ of distilled water), group $\mathrm{B}$ - positive control (noise stress at $100 \mathrm{~dB}$ for $4 \mathrm{~h} / 15$ day +1 $\mathrm{mL} / \mathrm{kg}$ of distilled water), and group $\mathrm{C}$ (noise stress at $100 \mathrm{~dB}$ for $4 \mathrm{~h} / 15$ day $+0.04 \mathrm{mg} / \mathrm{kg}$ of selenium-yeast via intragastric route. Noise was produced by a $15 \mathrm{~W}$ loudspeakers driven by a white noise generator. The noise was perceived at $100 \mathrm{~dB}$ throughout the cage as monitored by a sound-level meter D2023 (S.NO-F02199: Cygnet Systems, Gurgaon, Haryana, India). The cognitive test was conducted on pregnancy day 18 and 19. Two types of cognitive tests were performed, namely, the novel object recognition test that evaluates working memory and Y-maze for spatial memory test.

\subsection{Novel Object Recognition Test}

The test was conducted according to the method of [11] with a slight modification; using an open field box made of $50 \mathrm{~cm} \times 50 \mathrm{~cm} \times 50 \mathrm{~cm}$. The rats were trained by allowing they explore the open field arena for $10 \mathrm{~min}$ prior to the testing day in the presence of two identical sample objects. The objects discriminated were made of wood distinguished in color and shape. In between the tests, the objects were cleaned with $10 \%$ ethanol solution to prevent olfactory cues. In the retention session, the rats were placed back in the same field, where one of the familiar objects was replaced by a novel object, and the rats were allowed to explore for $5 \mathrm{~min}$. Exploration in this case is define as the directing of rats nose toward the object at a distance of 2 $\mathrm{cm}$ or less. It excludes climbing and leaning on the objects.

The total exploration time of the familiar and novel objects were used to calculate 
discrimination index. The discrimination index is an index of measures of discrimination between the familiar and the novel objects corrected for exploratory activity. It is calculated as: (time spent on novel object - time spent on familiar object)/ (time spent on novel object + time spent on familiar object). The discrimination index ranges from -1 to 1 , with -1 indicating complete preference for the familiar object, 0 indicating no preference for either object, and 1 indicating complete preference for the novel object [12].

\subsection{Y-Maze Test}

$Y$ Maze is a behavioral test for measuring the willingness of rodents to explore new environments. Rodents typically prefer to investigate a new arm of the maze rather than returning to the one they previously visited. Many parts of the brain namely, hippocampus, septum, basal forebrain, and prefrontal cortex are involved in this task [13].

The Y-shaped maze has three arms at a $120^{\circ}$ angle from each other $(50 \times 50 \times 50 \mathrm{~cm})$. In this study, the working memory was assessed based on the existing protocols from previous literature [13]. The rat was placed at the center of the maze, and allowed to freely explore the three arms for 8 mins. The number of times the rats enter into each arm was counted; this is when all the four limbs are inside the arm. The number of arm entries and the number of triads or alternation (a triad when it visited all 3 arms consecutively) was used to calculate the percentage of alternation; which is a measure of working memory, the number of maximum spontaneous alternations is then the total number of arms entered, and the percentage alternation is calculated as (actual alternations /maximum alternations) $\times 100$. If a rat scored significantly above $50 \%$ alternations, these indicate a functional working memory. Note, after each trial, the $Y$ maze was cleaned with 5\% ethanol that prevented olfactory cues.

\subsection{Data Analysis}

GraphPad Prism 8.4 (San Diego, California, USA) was the statistical package used. The results were expressed in mean $( \pm$ SEM). And values of $(P<0.05)$ were considered statistical significant. One-way ANOVA was used to identify the difference between mean, followed by Tukey's post-hoc test.

\section{RESULTS}

\subsection{The Effects of Selenium-yeast on the Percentage Alternation in Pregnant Dams Exposed to Noise Stress Using $Y$ maze for Spatial Memory Test}

The Percentage alternation above $50 \%$ is an indicator of spatial memory retention and below $50 \%$ indicates memory deficit. Fig. 1 shows a significant increase $(P=.05)$ in Group A (58.7 \pm 4.360$)$, when compared with Group B (24.37 \pm 2.37$)$. However, there was no significant difference in Group A when compared with Group C (56.3 \pm 3.48$)$. Though a statistical $(P=$ $.05)$ increase was observed in Group $C$ the selenium-yeast administered group when compared with Group B noise stresses without antioxidant administration.

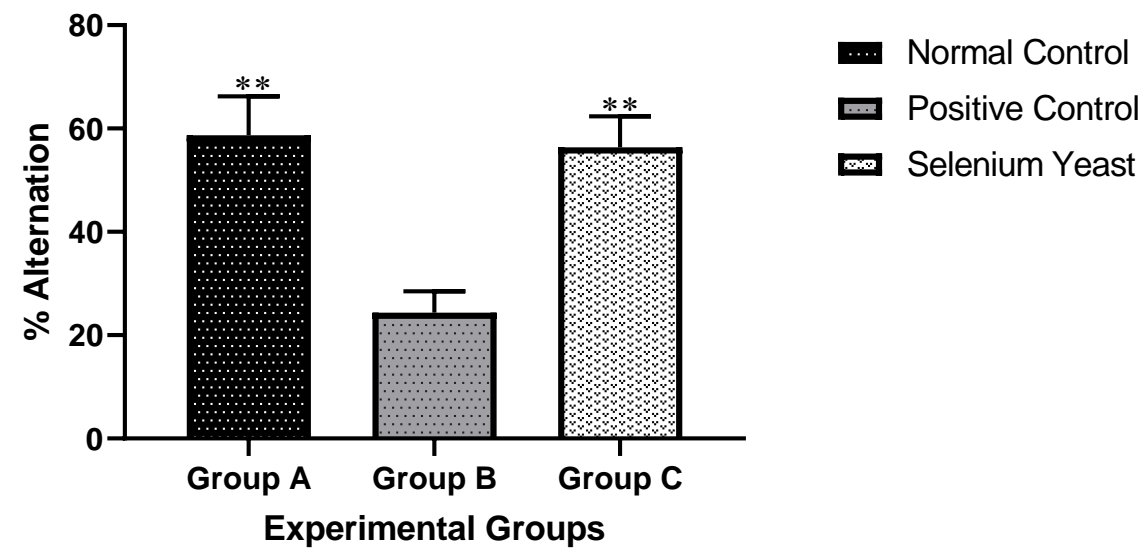

Fig. 1. The effects of selenium-yeast on the percentage alternation $n=4, \square \square$ indicates the degree of significant $(P=.05)$ 


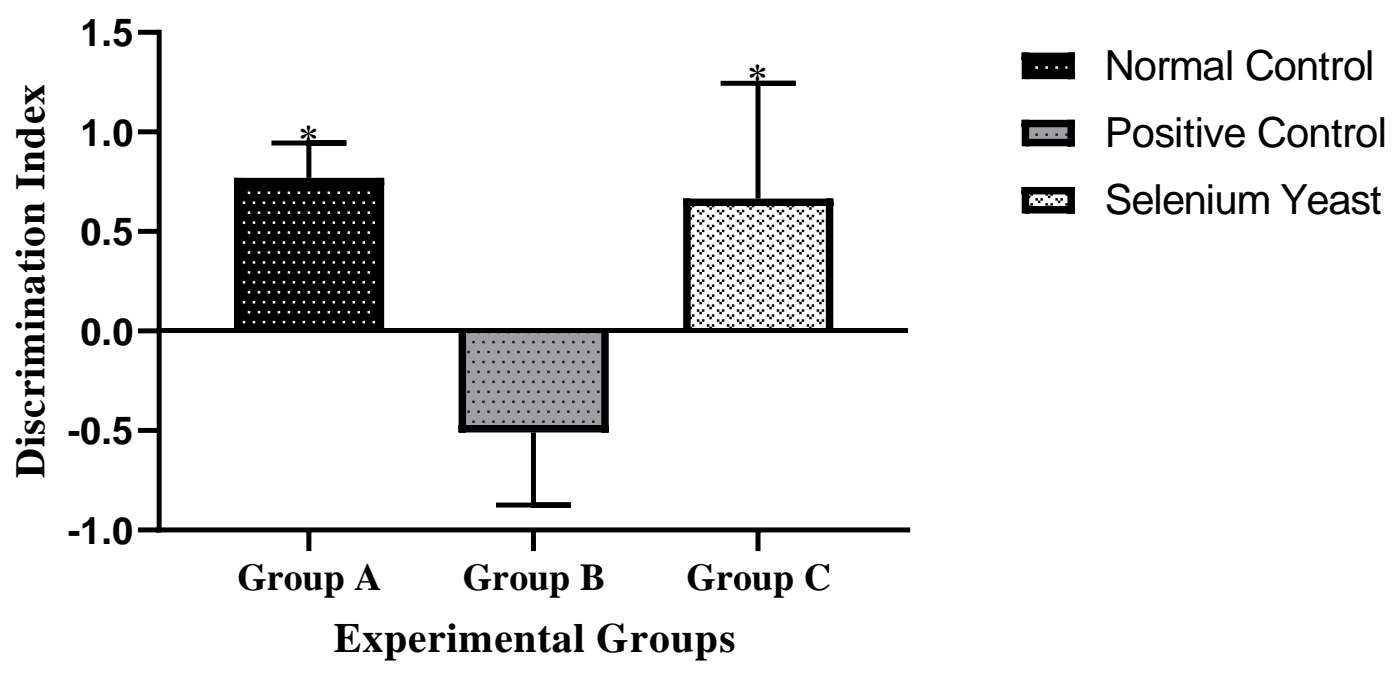

Fig. 2. The effects of selenium-yeast on discrimination index $n=4, \square$ indicate degree of significant difference $(P<0$.05)

\subsection{The Effects of Selenium-yeast on Discrimination Index in Pregnant Dams Exposed to Noise Stress Using Novel Object Recognition Test (NORT)}

Discrimination index (DI), range from $-1,0$ to 1 , with -1 indicating complete preference for the familiar object, 0 indicating no preference for either object, and 1 indicating complete preference for the novel object recognition (NOR), since rodents naturally want to explore new things, hence scoring 1 indicates retentive memory. Fig. 2 shows a statistical increase $(P=$ $.05)$ in $\mathrm{DI}$ of Group A $(0.77 \pm 0.11)$ when compared with Group B $(-0.51 \pm 0.21)$. There is a significant increase $(P=.05)$ in DI in Group C $(0.67 \pm 0.33)$ when compared with Group B.

\section{DISCUSSION}

Noise stress is an inescapable phenomenon in modern era, since we are been faced with it from multiple sources from the environment, but the adverse effects of this stress differs among gender, and this tends to be exaggerated in pregnant state [5]. Some studies have linked noise stress with several neurological disorders in both human and rodents [3,4], despite these findings no effective measure have been established to halt this effect. Fig. 1 shows an increases in percentage alternation in the nonstress group when compared with the stress group on the NOR test, since decrease alternation below $50 \%$ indicates impaired spatial memory; hence this study agrees with previous studies that shows noise stress impairs cognition $[14,15,16]$. However, there was an increase in the percentage alternation in selenium-yeast administered group exposed to noise stress, this finding agrees with previous studies that have reported the role of selenium-yeast as a cognitive booster $[17,18]$, this might be as a result of the antioxidants property of selenium-yeast in mitigating oxidative damages [18]. In Fig. 2, the discrimination index in the normal control group was significantly higher when compared with the stress control. The increase in discrimination index in group A indicates that rat not exposed to noise stress have a stable cognition, when compared with the stressed counterpart using the NORT; this study further buttress other report on noise stress inducing cognitive impairments $[16,19]$. However, the pre-administration of selenium-yeast prior to noise stress exposure was able to mitigate the negative effect that noise stress have on the cognition of pregnant dams which agrees with previous studies $[17,20]$ on the effect of selenium-yeast on memory. This study investigates the effects of selenium-yeast on the cognitive performance of the pregnant dams exposed to noise stress.

\section{CONCLUSION}

In conclusion, the results from this study show that noise stress induces cognitive impairments in pregnant dams on $Y$ maze and novel object 
recognition test in Wistar rats. However, the impairment caused through noise stress can be mitigated with the pre-administration of seleniumyeast.

\section{DISCLAIMER}

The products used for this research are commonly and predominantly use products in our area of research and country. There is absolutely no conflict of interest between the authors and producers of the products because we do not intend to use these products as an avenue for any litigation but for the advancement of knowledge. Also, the research was not funded by the producing company rather it was funded by personal efforts of the authors.

\section{ETHICAL APPROVAL}

The ethical clearance was obtained from the Baze University Abuja Animal Ethical Committee.

\section{ACKNOWLEDGEMETS}

I will like to appreciate Dr. Uche Kachi and also Mr. Ojo Kafaru for his technical assistance.

\section{COMPETING INTERESTS}

Authors have declared that no competing interests exist.

\section{REFERENCES}

1. Singh N, Davar, SC. Noise pollutionsources, effects and control. J of Hum Eco. 2004;16(3):181-187.

2. Basner $M$, Babisch W, Davis A, Brink M, Clark C, Janssen S, Stansfeld, S. Auditory and non-auditory effects of noise on health. The lancet, 2014;383(9925):13251332.

3. Lercher P, Evans GW, Meis, M. Ambient noise and cognitive processes among primary schoolchildren. Env Behav. 2003;35(6):725-735.

4. Azman KF, Zakaria R, AbdAziz C, Othman Z, Al-Rahbi B. Tualang honey improves memory performance and decreases depressive-like behavior in rats exposed to loud noise stress. Noi Health. 2015;17(75):83.

5. Laulhé $M$, Dumeige L, Vu TA, Hani I, Pussard $E$, Lombès $M$, Martinerie $L$. Sexual dimorphism of corticosteroid signaling during kidney development. Intern J Mol Sci. 2021;22(10):5275.

6. Vetrovoy $O$, Stratilov V, Nimiritsky $P$, Makarevich $\mathrm{P}$, Tyulkova E. Prenatal hypoxia induces premature aging accompanied by impaired function of the glutamatergic system in rat hippocampus. Neuro Res. 2021;46(3):550-563.

7. Wu D, Zhang S, Sun N, Zhu B, Lin S. Neuroprotective function of a novel hexapeptide QMDDQ from shrimp via activation of the PKA/CREB/BNDF signaling pathway and its structure-activity relationship. J Agric Food Chem. 2020; 68(24):6759-6769.

8. Motta JR, Jung IEDC, Azzolin VF, Teixeira CF, Braun LE, De Oliveira Nerys DA, Barbisan, F. Avocado oil (Persea americana) protects SH-SY5Y cells against cytotoxicity triggered by cortisol by the modulation of BDNF, oxidative stress, and apoptosis molecules. J Food Biochem. 2021;45(2):e13596.

9. Atasoy N, Yücel UM. Antioxidants from plant sources and free radicals; 2021.

10. Jia X, Li J, Li S, Zhao Q, Zhang K, Tang C, Qin Y. Effects of dietary supplementation with different levels of selenium yeast on growth performance, carcass characteristics, antioxidant capacity, and meat quality of Tan sheep. Livestock Sci. 2022;255:104783.

11. Hendrickx JO, De Moudt S, Calus E, De Deyn PP, Van Dam D, De Meyer GR. Agerelated cognitive decline in spatial learning and memory of C57BL/6J mice. Behav. Brain Res. 2022;418:113649.

12. Zhang R, Xue G, Wang S, Zhang L, Shi C, Xie $X$. Novel object recognition as a facile behavior test for evaluating drug effects in AßPP/PS1 Alzheimer's disease mouse model. J Alz Dis. 2012;31(4):801-812.

13. Kilari EK, Rao LSN, Sreemanthula S, Kola PK. Anti-stress and nootropic activity of aqueous extract of Piper longum fruit, estimated by noninvasive biomarkers and Y-maze test in rodents. Environ Exp Biol. 2015;13:25-31.

14. Gheewalla F, McClelland A, Furnham, A. Effects of background noise and extraversion on reading comprehension performance. Ergon. 2021;64(5):593-599.

15. Jafari Z, Okuma M, Karem H, Mehla J, Kolb BE, Mohajerani MH. Prenatal noise stress aggravates cognitive decline and the onset and progression of beta amyloid 
pathology in a mouse model of Alzheimer's disease. Neuro Aging. 2019;77:66-86.

16. Jafari Z, Kolb BE, Mohajerani MH. Noise exposure accelerates the risk of cognitive impairment and Alzheimer's disease: Adulthood, gestational, and prenatal mechanistic evidence from animal studies. Neurosci. Biobehav. Rev. 2020;117:110128.

17. Zhang ZH, Wen L, Wu QY, Chen C, Zheng $\mathrm{R}$, Liu $\mathrm{Q}$, Song GL. Long-term dietary supplementation with selenium-enriched yeast improves cognitive impairment, reverses synaptic deficits, and mitigates Tau pathology in a triple transgenic mouse model of Alzheimer's disease. J Agric. Food Chem. 2017;65(24):4970-4979.

18. Song G, Zhang Z, Wen L, Chen C, Shi Q, Zhang Y, Liu Q. Selenomethionine ameliorates cognitive decline, reduces tau hyperphosphorylation, and reverses synaptic deficit in the triple transgenic mouse model of Alzheimer's disease. J Alz Dis. 2014;41(1):85-99.

19. Moore SJ, Deshpande K, Stinnett GS, Seasholtz AF, Murphy GG. Conversion of short-term to long-term memory in the novel object recognition paradigm. Neuro Learning and Memory. 2013;105: 174-185.

20. Asl SS, Amiri I, Samzadeh-Kermani A, Abbasalipourkabir R, Gholamigeravand B, Shahidi S. Chitosan-coated Selenium nanoparticles enhance the efficiency of stem cells in the neuroprotection of streptozotocin-induced neurotoxicity in male rats. The Inter J. Biochem. Cell Bio. $2021 ; 141: 106089$.

(C) 2022 Ochayi et al.; This is an Open Access article distributed under the terms of the Creative Commons Attribution License (http://creativecommons.org/licenses/by/4.0), which permits unrestricted use, distribution, and reproduction in any medium, provided the original work is properly cited. https://www.sdiarticle5.com/review-history/81810 